\title{
El Término Glía, una Tradición Conceptual Errada: Propuesta de Cambio por Sinneuronas
}

\author{
The Term Glia, A Wrong Conceptual Tradition: Proposal for Change for Synneurons
}

\author{
Jorge Eduardo Duque Parra ${ }^{1,3,4}$; John Barco Ríos ${ }^{1,3}$ \& Johanna Alexandra Barco Cano ${ }^{2,3}$
}

DUQUE, P. J. E.; BARCO, R. J. \& BARCO, C. J. A. El término glía, una tradición conceptual errada: propuesta de cambio por sinneuronas. Int. J. Morphol., 39(2):638-641, 2021.

RESUMEN: Desde su descubrimiento, las células no neuronales del sistema nervioso recibieron el nombre de glia, palabra de origen griego que significa unión o pegamento, porque se creía que su función era formar una especie de masilla en la que se encuentran inmersas las neuronas. Desde entonces, mediante nuevas técnicas de tinción, se descubrieron otros tipos celulares que fueron catalogados también como glía, que hasta la fecha siguen siendo consideradas como las células de unión o pegamento del tejido nervioso. El objetivo de este artículo es cuestionar el uso inadecuado del término glía y proponer un nuevo término para designar a las células no neuronales. A pesar del enorme conocimiento que actualmente se tiene de estas células y de la gran variedad de funciones que realizan para mantener el correcto funcionamiento de las neuronas y los circuitos nerviosos, aún se les conserva el nombre de glía, un término errado que desdibuja el verdadero papel que cumplen y su importancia para el sistema nervioso. Por lo anterior, se propone el término "sinneuronas", del prefijo griego syn que significa con o junto con, lo que daría a entender que son células que presentan cercanía estructural y funcional con las neuronas.

PALABRAS CLAVE: Historia; Neuroglía; Sistema nervioso; Terminología (DeCS).

\section{INTRODUCCIÓN}

"Si la verdad, si todas las verdades no están sujetas al examen del "ensayo y el error", si no existe una libertad que permita a los hombres cuestionar y compulsar la validez de todas las teorías que pretenden dar respuesta a los problemas que enfrentan, la mecánica del conocimiento se ve trabada y éste puede ser pervertido. Entonces, en lugar de verdades racionales, se entronizan mitos, actos de fe, magia." * Mario Vargas Llosa. Premio Nobel de literatura. 2010.

Se considera que fue el neuroanatomista alemán Otto Friedrich Karl Deiters (1834-1863) (Deiters \& Guillery, 2013) el primero en identificar en el sistema nervioso central la presencia de un tipo de células que no tenían ningún parecido a las neuronas, porque, según él, no presentaban axón, por lo que más adelante recibieron el nombre de células de Deiters en su honor. Sin embargo, es a Rudolph Ludwig Karl Virchow (1821-1902) a quien se le atribuye el honor de ser el descubridor de la glía, cuando propuso la idea que debajo de la capa de células ependimarias que revisten los ventrículos cerebrales debía haber un revestimiento a manera de lámina de tejido conectivo (Kattenmann \& Ransom, 2012). En este sentido, Virchow era contrario a la opinión de otros investigadores, como Jean Evangelista Purkinje (1787-1869), quien decía que el cerebro no contenía ningún tejido conectivo y que el epéndimo no era más que una capa de epitelio (Somjen, 1988). En su libro titulado Patología Celular, publicado en 1858, Virchow describe que en el cerebro hay otra clase de células con forma de huso y estrellada distinta a las neuronas y que hacen parte del tejido conectivo, por lo que les dio el nombre de nervenkitt y posteriormente glía. Nervenkitt es una palabra alemana que significa "pegamento o cemento nervioso", queriendo decir con ello que tales células forman un tipo de masilla en la que están inmersas las neuronas (Virchow, 1856). Mientras que el término glia procede del griego $\gamma \lambda \iota \alpha$, que significa liga, unión o pegamento (Jäkel \& Dimou, 2017; Purves et al., 2008), como un tejido de embalaje para las neuronas pero

\footnotetext{
${ }^{1}$ Universidad de Caldas, Facultad de Ciencias para la Salud, Departamento de Ciencias Básicas, Manizales, Colombia.

${ }^{2}$ Universidad de Caldas, Facultad de Ciencias para la salud, Departamento Clínico, Manizales, Colombia.

${ }^{3}$ Universidad de Caldas, Departamento de Ciencias Básicas, Grupo Neurociencia de Caldas, Manizales, Colombia.

${ }^{4}$ Universidad de Manizales, Facultad de Ciencias para la Salud, Departamento de Ciencias Básicas, Manizales, Colombia.
} 
que no son fibroblastos (Duque Parra, 2014). Además de Virchow, otros investigadores también observaron nuevos tipos celulares que hacían parte del sistema nervioso y contribuyeron con sus ideas al conocimiento de la glía. Por ejemplo, Theodor Schwann (1810-1882) identificó las células que acompañan y rodean las fibras nerviosas del sistema nervioso periférico, y posteriormente se determinó que forman las vainas de mielina (Aldskogius \& Fraher, 2002). Camilo Golgi (1843-1926), mediante un método de tinción celular que ideó a base de bicromato de potasio y nitrato de plata, conocida como técnica de impregnación argéntica, pudo observar la morfología neuronal completa en tres dimensiones (Torres-Fernández, 2006) y la organización estructural que adopta el tejido nervioso (López-Muñoz et al., 2006), pero además describió las características estructurales de las células gliales y corroboró lo que años antes había dicho Deiters, que estas células no neuronales mostraban una morfología compleja y carecían de axón. La imagen de una de estas células fue publicada por Golgi y luego se comprobó que se trataba de un oligodendrocito.

Con algunos pequeños cambios que le hizo al método de coloración de Golgi, entre ellos la doble impregnación argéntica (Torres-Fernández), Santiago Ramón y Cajal (1852-1934) pudo interpretar sus preparaciones histológicas y realizó dibujos precisos de la estructura y organización neuronal (Palacios et al., 2015). Además, en 1913, describió en detalle la morfología de los astrocitos y la relación que establecen con las neuronas y los pequeños vasos sanguíneos. Posteriormente, Pio del Río Hortega (1882-1945) estudió otros elementos nerviosos de naturaleza desconocida que Cajal había observado previamente y les había dado el nombre de corpúsculos apolares; con un método de coloración que él mismo desarrolló a base de carbonato de plata amoniacal (Iglesias-Rozas \& Garrosa, 2013), del Río Hortega demostró que tales elementos incluían dos tipos celulares distintos, las microglías (conocidas por los eponimistas como células de Hortega) y los oligodendrocitos, y después realizó un estudio completo de su morfología, función e histogénesis de ambas células (Pérez-Cerdá et al., 2015).

Con el desarrollo de nuevas técnicas de microscopía, los neurohistólogos han descubierto otros tipos de células no neuronales inmersas en el sistema nervioso, cada una de ellas con funciones muy particulares. No obstante, su nominación inicial de glía se conserva hasta nuestros días y en la Terminologia Anatomica aparecen referenciadas con el nombre de "neuroglia", con el código A14.0.00.005 (Federative Committee on Anatomical Terminology, 1998), mientras que en la Terminologia Histologica figura con el código H2.00.06.2.00001 (Federative International Committee on Anatomical Terminology, 2008).

\section{DISCUSIÓN}

En numerosos libros de texto y artículos de neurología en los que hacen referencia a las células gliales, es frecuente encontrar que les asignan una función general como soporte estructural del tejido nervioso (García-Porrero \& Hurlé, 2015). Incluso, se insiste que las células de sostén de las neuronas son específicamente los astrocitos y los pericitos (Hillman, 2015). Esta generalización ha originado una falsa idea sobre la real función que realiza cada una de las células gliales, pues da a entender que todas ellas tienen la capacidad intrínseca de producir y secretar ciertas proteínas que actúan como material de cemento para el anclaje de las neuronas adyacentes y brindarles un soporte estructural (García-Porrero \& Hurlé). Sin embargo, distintos estudios han revelado que solamente los neurolemocitos (o células de Schwann) tienen la capacidad de expresar colágeno tipo $\mathrm{V}$ que contiene una cadena a4 (Chernousov et al., 2000; Erdman et al., 2002), y que dicha molécula posiblemente cumple una función importante en el desarrollo del sistema nervioso periférico (Chernousov et al.). Se cree que este colágeno a4 (V) participa en la regeneración nerviosa periférica, y que la incapacidad de regeneración que muestra el sistema nervioso central después de una lesión es consecuencia del medio molecular (Erdman et al.) y de la ausencia de colágeno.

La generalización errada que se hace de las células gliales como sostén del tejido nervioso se ha mantenido prácticamente desde su descubrimiento como un dogma más de la neurología, a pesar de todo el conocimiento que se tiene actualmente de dichas células y de las variadas funciones que realizan (Dimou \& Götz, 2014). Los astrocitos, por ejemplo, son funcionalmente muy versátiles, puesto que se encargan de formar la barrera hematoencefálica para regular el paso de ciertas moléculas hacia las neuronas y modulan además el flujo sanguíneo cerebral (Duque Parra); tienen la capacidad de almacenar energía metabólica en forma de glucógeno; participan activamente en la plasticidad sináptica removiendo aquellas sinapsis inoperantes y facilitando la formación de nuevos contactos entre neuronas (Kim et al., 2017); liberan algunos neurotransmisores y además eliminan el exceso de ellos de las hendiduras sinápticas, así como del potasio que liberan las neuronas al medio extracelular (Tamayo Orrego \& Duque Parra, 2007), producen factores tróficos necesarios para la supervivencia de las neuronas y para mantener la plasticidad sináptica (Fields, 2008; Dossi et al., 2018); reaccionan frente a una isquemia cerebral transitoria transformándose en astrocitos reactivos, los cuales actúan como fagocitos para eliminar restos celulares (Morizawa et al., 2017), y combaten también contra agentes patógenos liberando citoquinas para atraer a las células inmunitarias. 
Por otra parte, los oligodendrocitos tienen como función primordial la mielinización de las fibras nerviosas en el sistema nervioso central con el fin de permitir la propagación rápida de los impulsos nerviosos (Nave \& Werner, 2014), mientras que esta misma función la desempeñan los neurolemocitos en el sistema nervioso periférico, pero además juegan un papel importante en la regeneración de las fibras nerviosas (Kim et al., 2016; Feltri et al., 2016). Las microglías son consideradas como los fagocitos profesionales del cerebro, pues son los encargados de eliminar rápidamente los microorganismos patógenos y remover los desechos apoptóticos de neuronas muertas para evitar sus efectos tóxicos (Diaz-Aparicio et al., 2016). Los ependimocitos y los tanicitos hacen parte del epitelio ependimario que tapiza internamente las cavidades ventriculares del encéfalo y el conducto central de la médula espinal (Duque Parra et al., 2017). En algunas regiones los ependimocitos son ciliados con el propósito de facilitar el movimiento del líquido cerebroespinal, mientras que en otras regiones regulan el transporte de iones, agua y moléculas pequeñas entre el líquido cerebroespinal y el tejido nervioso (Bruni, 1998). Los tanicitos son células ependimarias especializadas que se localizan entre el tercer ventrículo y la eminencia media hipotalámica, y se cree que se encargan de controlar la exposición a señales que vienen de la sangre actuando como sensores y reguladores de la entrada y salida hipotalámica (Rizzoti \& Lovell-Badge, 2017).

La glía radial cumple una función importante durante el desarrollo del sistema nervioso central, pues forman una especie de andamiaje fibroso sobre el cual las neuronas recién formadas migran a sus destinos finales en la corteza cerebral (Rakic, 2009). Una vez concluida la migración neuronal, la glía radial migra también a la superficie de la corteza y se transforman en astrocitos y oligodendrocitos (Kriegstein \& Alvarez-Buylla, 2009; Mo \& Zecevic, 2009). Las células glial satélites, se encargan de formar una cápsula alrededor de los ganglios espinales, craneales y viscerales, donde participan activamente en el procesamiento de la información sensorial al interactuar con los somas neuronales (Huang et al., 2013) formando una unidad funcional; las evidencias también apuntan a que dichas células controlan el microambiente neuronal (Hanani, 2010).

Como se puede apreciar, la neuroglia no se limita a cumplir un supuesto apoyo mecánico de las neuronas (Duque Parra) como se planteó inicialmente, sino que cumplen un amplio espectro de funciones que son fundamentales para el correcto funcionamiento de las neuronas y el mantenimiento de los circuitos nerviosos (Argente-Arizón et al., 2017). Por lo tanto, el término glía de origen griego, que significa liga, unión o pegamento (Jäkel \& Dimou; Purves et al.), no es el nombre apropiado para designar a estas célu- las, porque induce a pensar erróneamente en una función que, a la luz de los conocimientos actuales, no le corresponde. Creemos que el nombre más apropiado para designarlas es el de "synneuronas", con el prefijo griego "syn" que significa "con, conjuntamente, junto con" (Monlau, 1856), lo que denotaría que son células nerviosas no neuronales que acompañan a las neuronas y contribuyen a su funcionalidad.

Los nombres empleados en la terminología médica guardan una información intrínseca que permite describir con precisión la estructura o concepto que se desea representar. No obstante, algunos términos no reflejan del todo esta información o fueron creados cuando apenas se tenía poco conocimiento de su estructura y función, como sucedió en el caso de la glía, un nombre que se ha mantenido en el vocabulario médico por tradición dogmática aunque su significado no represente para nada el verdadero papel que realizan. La ciencia per se es dinámica y renovadora, pero la naturaleza humana gusta de la tradición y el dogmatismo.

\section{CONCLUSIÓN}

El término griego glía, que significa unión o pegamento, no refleja la naturaleza funcional de las células nerviosas que no son neuronas porque ellas realmente no pegan nada. Estas células no neuronales cumplen una gran variedad de funciones que tienen como propósito mantener el correcto funcionamiento de las neuronas y los circuitos nerviosos que originan. Por lo anterior, consideramos que el nombre más apropiado para estas células es el de "sinneuronas", debido a su cercanía estructural y funcional con las neuronas. La tradición y el dogmatismo son conceptos que van en contraposición con la ciencia; por tanto, proponemos abolir el tradicional término neuroglia de las terminologías anatómica e histológica.

DUQUE, P. J. E.; BARCO, R. J. \& BARCO, C. J. A. The term glia, a wrong conceptual tradition: proposal for change for synneurons. Int. J. Morphol., 39(2):638-641, 2021.

SUMMARY: Since their discovery, the non-neuronal cells of the nervous system have been called glia, a word of Greek origin that means union or glue, because it was believed that their function was to form a kind of putty, in which neurons are immersed. Thereafter, new cell types discovered by new staining techniques, were also classified as glia, which to this day are still considered as binding cells or glue of nerve tissue. The objective of this paper is to question the inappropriate use of the term glia and to propose a new term to designate non-neuronal cells. Despite the enormous knowledge that is currently available of these cells and the great variety of functions they perform to maintain the proper functioning 
of neurons and nerve circuits, they still retain the name of glia, an inappropriate name that blurs the true role they play. Therefore, the term "synneuronas" is proposed, from the Greek prefix syn which means with or together with, what would suggest that they are cells that present structural and functional proximity with to neurons.

\section{Terminology (DeCS).}

KEY WORDS: History; Neur oglia; Nervous system;

\section{REFERENCIAS BIBLIOGRÁFICAS}

Aldskogius, H. \& Fraher, J. Glial Interfaces in the Nervous System. Role in Repair and Plasticity. Amsterdam, IOS Press, 2002.

Argente-Arizón, P.; Guerra-Cantera, S.; García-Segura, L. M.; Argente, J. \& Chowen, J. A. Glial cells and energy balance. J. Mol. Endocrinol., 58(1):R59-71, 2017.

Bruni, J. E. Ependymal development, proliferation, and functions: a review. Microsc. Res. Tech., 41(1):2-13, 1998.

Chernousov, M. A.; Rothblum, K.; Tyler, W. A.; Stahl, R. C. \& Carey, D. J. Schwann cells synthesize type V collagen that contains a novel alpha 4 chain. Molecular cloning, biochemical characterization, and high affinity heparin binding of alpha 4(V) collagen. J. Biol. Chem., 275(36):28208$1,2000$.

Deiters, V. S. \& Guillery, R. W. Otto Friedrich Karl Deiters (1834-1863). J. Comp. Neurol., 521(9):1929-53, 2013.

Diaz-Aparicio, I.; Beccari, S.; Abiega, O. \& Sierra, A. Clearing the corpses: regulatory mechanisms, novel tools, and therapeutic potential of harnessing microglial phagocytosis in the diseased brain. Neural. Regen. Res., 11(10):1533-9, 2016.

Dimou, L. \& Götz, M. Glial cells as progenitors and stem cells: new roles in the healthy and diseased. Brain. Physiol. Rev., 94:709-37, 2014.

Dossi, E.; Vasile, F. \& Rouach, N. Human astrocytes in the diseased brain. Brain Res. Bull., 136:139-56, 2018.

Duque Parra, J. E. El Tejido Glial. Las Células Nerviosas que No Son Neuronas. Manizales, Universidad Autónoma de Manizales, 2014.

Duque Parra, J. E.; Barco Ríos, J. \& García Aguirre, J. F. A historical approach to the ventricular system of the brain. Rev. Fac. Med., 65(3):473-7, 2017.

Erdman, R.; Stahl, R. C.; Rothblum, K.; Chernousov, M. A. 6 Carey, D. J. Schwann Cell Adhesion to a Novel Heparan Sulfate Binding Site in the $\mathrm{N}$-terminal Domain of a4 Type V collagen is mediated by syndecan-3. J. Biol. Chem., 277(9):7619-25, 2002.

Federative Committee on Anatomical Terminology (FCAT). Terminologia Anatomica: International Anatomical Terminology. Stuttgart, Georg Thieme Verlag, 1998.

Federative International Committee on Anatomical Terminology (FICAT). Terminologia Histologica: International Terms for Human Cytology and Histology. Philadelphia, Wolters Kluwer/Lippincott Williams \& Wilkins, 2008.

Feltri, M. L.; Poitelon, Y. \& Previtali, S. C. How schwann cells sort axons: new concepts. Neuroscientist, 22(3):252-5, 2016.

Fields, D. White matter. Sci. Am., 298:54-61, 2008.

García-Porrero, J. \& Hurlé, J. M. Neuroanatomía humana. Buenos Aires, Médica Panamericana, 2015.

Hanani, M. Satellite glial cells in sympathetic and parasympathetic ganglia: in search of function. Brain Res. Rev., 64(2):304-27, 2010.

Hillman, E. M. C. El flujo sanguíneo en el cerebro. Mente Cereb., 75:6471, 2015.

Huang, L. Y.; Gu, Y. \& Chen, Y. Communication between neuronal somata and satellite glial cells in sensory ganglia. Glia, 61(10):1571-81, 2013.

Iglesias-Rozas, J. R. \& Garrosa, M. Rio-Hortega's Third Contribution to the Morphological Knowledge and Functional Interpretation of the Oligodendroglia. New York, Elsevier, 2013.
Jäkel, S. \& Dimou, L. Glial cells and their function in the adult brain: a journey through the history of their ablation. Front. Cell. Neurosci., 11:24, 2017.

Kattenmann, H. \& Ransom, B. R. Neuroglia. 3a ed. New York, Oxford University Press, 2012.

Kim, S. K.; Nabekura, J. \& Koizumi, S. Astrocyte-mediated synapse remodeling in the pathological brain. Glia, 65(11):1719-27, 2017.

Kim, S.; Maynard, J. C.; Sasaki, Y.; Strickland, A.; Sherman, D. L.; Brophy, P. J.; Burlingame, A. L. \& Milbrandt, J. Schwann cell O-GlcNAc glycosylation is required for myelin maintenance and axon integrity. $J$. Neurosci., 36(37):9633-46, 2016.

Kriegstein, A. \& Alvarez-Buylla, A. The glial nature of embryonic and adult neural stem cells. Annu. Rev. Neurosci., 32:149-84, 2009.

López-Muñoz, F.; Álamo, C.; García-García, P. \& Boya, J. Relevancia histórica de la teoría neuronal un siglo después del Nobel de Cajal: implicaciones psiquiátricas y psicofarmacológicas. Psiq. Biol., 13(5):16782, 2006.

Mo, Z. \& Zecevic, N. Human fetal radial glia cells generate oligodendrocytes in vitro. Glia, 57(5):490-8, 2009.

Monlau, P. F. Diccionario Etimológico de la Lengua Castellana (Ensayo). Madrid, Imprenta y Estereotipia de M Rivadeneyra, 1856.

Morizawa, Y. M.; Hirayama, Y.; Ohno, N.; Shibata, S.; Shigetomi, E.; Sui, Y.; Nabekura, J.; Sato, K.; Okajima, F.; Takebayashi, H.; et al. Reactive astrocytes function as phagocytes after brain ischemia via ABCA1mediated pathway. Nat. Commun., 8(1):28, 2017.

Nave, K. A. \& Werner, H. B. Myelination of the nervous system: mechanisms and functions. Annu. Rev. Cell Dev. Biol., 30:503-33, 2014.

Palacios, S. L.; Vergara, M. L. D.; Liévano, J. P. \& Guerrero, A. Santiago Ramón y Cajal, neurocientífico y pintor. Acta Neurol. Colomb., 31(4):45461, 2015.

Pérez-Cerdá, F.; Sánchez-Gómez, M. V. \& Matute, C. Pío del Río Hortega and the discovery of the oligodendrocytes. Front. Neuroanat., 9:92, 2015.

Purves, D.; Augustine, G. J.; Fitzpatrick, D. \& Klajn, D. S. Neurociencia. 3a ed, Madrid, Médica Panamericana, 2008.

Rakic, P. Evolution of the neocortex: a perspective from developmental biology. Nat. Rev. Neurosci., 10(10):724-35, 2009.

Rizzoti, K. \& Lovell-Badge, R. Pivotal role of median eminence tanycytes for hypothalamic function and neurogenesis. Mol. Cell. Endocrinol., 445:7-13, 2017.

Somjen, G. G. Nervenkitt: notes on the history of the concept of neuroglia. Glia, 1(1):2-9, 1988.

Tamayo Orrego, L. \& Duque Parra, J. E. Regulación metabólica de la microcirculación cerebral. Rev. Neurol., 44(7):415-25, 2007.

Torres-Fernández, O. La técnica de impregnación argéntica de Golgi. Conmemoración del centenario del premio nobel de Medicina (1906) compartido por Camilo Golgi y Santiago Ramón y Cajal. Biomédica, 26(4):498-508, 2006.

Virchow, R. Über das granulierte Ansehen der Wandungen der Gerhirnventrikel. Allg. Z. Psychiatr., 3:424-50, 1856.

Dirección para correspondencia:

Jorge Eduardo Duque Parra

Universidad de Caldas

Sede Principal Calle 65 \# 26-10

oficina: M203.

Manizales

COLOMBIA

E-mail: jduqueparra@yahoo.com.mx

Recibido : 29-11-2020

Aceptado: 14-01-2021 